\title{
La protection des programmes de vaccination et du public
}

$\mathrm{E}$ n I986, le journal publiait un article de la Société canadienne de pédiatrie préconisant que le Canada mette sur pied un programme d'indemnisation sans égard à la responsabilité pour les cas de complications de la vaccination ${ }^{1}$. Plus de deux décennies plus tard, il n'existe aucun système national de cette nature, en dépit des appels répétés à cet égard. À la lumière d'événements récents, y compris la nécessité possible d'administrer à des millions de Canadiens, contre la grippe pandémique, un vaccin dont les tests ne sont pas terminés et l'augmentation rapide du nombre de vaccins pédiatriques recommandés, la création d'un tel programme constitue toutefois une priorité nationale.

Le pilier d'un système d'indemnisation sans égard à la responsabilité, c'est qu'une personne est vaccinée non seulement pour son propre bien, mais aussi pour celui de la communauté. C'est pourquoi, si une personne est victime d'une complication alors qu'elle contribue à ce bien public, l'argument déontologique en faveur de l'indemnisation est solide. Ce processus d'indemnisation devrait contourner le système judiciaire, mal adapté à ce type de problème. Dans son rapport marquant sur la tragédie du sang contaminé, le juge Horace Krever préconisait un système d'indemnisation sans égard à la responsabilité pour les personnes infectées par les produits sanguins contaminés. Il soutenait que les personnes en cause, déjà lésées, ne devraient pas avoir à endurer le processus judiciaire pour obtenir réparation.

Au cours des années I980, les États-Unis ont mis en œuvre l'indemnisation sans égard à la responsabilité pour deux raisons autres que l'éthique et la justice sociale. Tout d'abord, une crise de confiance publique à l'égard de la vaccination a fait baisser les taux d'immunisation. Deuxièmement, des centaines de poursuites en justice intentées contre les fabricants de vaccins étaient en train de miner rapidement l'industrie. Beaucoup de fabricants ont mis fin à leur production, ce qui a causé des pénuries, fait grimper le coût des vaccins et refroidi l'innovation ${ }^{2}$. Le régime américain sans égard à la responsabilité oblige une personne à démontrer qu'elle a subi un effet indésirable reconnu dans un laps de temps précis après la vaccination. L'indemnisation repose alors sur des montants prédéterminés en fonction de l'étendue du traumatisme. En rétrospective, le lancement du programme sans égard à la responsabilité est considéré comme un succès et on lui attribue en partie le crédit d'avoir redonné au public confiance dans les vaccins et créé un environnement protégé pour les fabricants de vaccins - tout en indemnisant les personnes lésées.

Au Canada, cette crise de confiance à l'égard des vaccins n'existe pas pour le moment, et nous n'avons pas non plus les mêmes préoccupations au sujet des poursuites en justice contre nos fournisseurs de vaccins. D'importantes préoccupations commencent néanmoins à prendre forme. Le plus urgent peut-être, c'est que la vaccination, $\exists$ de toute nécessité rapide, en cas de grippe pandémique aura inévitablement pour effet d'exposer des gens à des vaccins dont on n'aura pas en le temps d'établir l'innocuité. Les fabricants pourront être réticents à distribuer de tels vaccins sans bénéficier d'une protection contre la responsabilité civile, et les gens pourront être réticents à recevoir un vaccin qui n'a pas été testé comme il se doit. Ces deux malaises mineraient les efforts de lutte contre la pandémie. Un programme d'indemnisation sans égard à la responsabilité pourrait dissiper ces deux préoccupations en protégeant légalement les fabricants et en tenant compte des préoccupations des personnes vaccinées. Deuxièmement, l'acceptation par le public du nombre croissant de vaccins infantiles recommandés pourrait un jour atteindre ses limites. Un programme d'indemnisation sans égard à la responsabilité pourrait aider à maintenir l'appui de la population envers ces vaccins à mesure que les programmes continuent de prendre de l'ampleur, en démontrant l'engagement du secteur de la santé publique envers les personnes vaccinées. Troisièmement, comme on met davantage de vaccins en service, la probabilité d'un effet indésirable rare non défini au cours d'essais cliniques s'accentue. Un programme d'indemnisation pourrait protéger les personnes qui ont subi un préjudice et aider ainsi à maintenir la confiance du public lorsque de tels épisodes se produisent.

Il existe des programmes d'indemnisation sans égard à la responsabilité dans aux moins $\mathrm{I}_{3}$ provinces ou pays, y compris les États-Unis et la province de Québec ${ }^{3}$. On a démontré que les programmes sont à la fois faisables et rentables. Le Parlement pourrait adopter une loi créant le programme sans soulever beaucoup de controverse. Les sommes payées en cas de complication et la façon de financer le système constituent bien entendu des détails d'une importance énorme, mais nous aurions la chance de tirer des leçons de l'expérience d'autres pays.

Il ne faut pas attendre que les problèmes qui ont déclenché l'adoption de l'indemnisation sans égard à la responsabilité aux États-Unis fassent leur apparition au Canada avant de lancer un tel programme. Une fois la confiance de la population perdue, il est difficile de la retrouver - et nos programmes de vaccination ont trop d'importance pour la protection de la santé publique pour courir ce risque. Le plus important demeure toutefois que le Canada devrait instaurer l'indemnisation sans égard à la responsabilité parce que c'est la façon juste et éthique de s'occuper des très rares cas où un vaccin a causé un préjudice.

\section{Kumanan Wilson}

Professeur agrégé

Département de médecine

Université de Toronto

Toronto (Ont.)

Pour l'Équipe de rédaction de l'éditorial (Paul C. Hébert, Matthew Stanbrook, Barbara Sibbald, Ken Flegel,

Noni McDonald et Amir Attaran)

Intérêts concurrents : Aucun déclaré.

\section{RÉFÉRENCES}

I. Société canadienne de pédiatrie. In support of a compensation plan for vaccineassociated injuries. JAMC 1986;135:747-9.

2. Evans G. Update on vaccine liability in the United States: presentation at the National Vaccine Program Office Workshop on strengthening the supply of routinely recommended vaccines in the United States, I2 février 2002. Clin Infect Dis 2006;42(Suppl 3):Sizo-7.

3. Evans G. Vaccine injury compensation programs worldwide. Vaccine i999; I7(Suppl 3):S25-35. 